\title{
SOME PROPERTIES OF FIBONACCI LANGUGAES
}

\author{
CHENG-MING FAN AND H. J. SHYR
}

\begin{abstract}
Two particular types of Fibonacci languages $F_{a, b}^{1}=\{a, b, a b, b a b, a b b a b$, $\ldots\}$ and $F_{a, b}^{0}=\{a, b, b a, b a b, b a b b a, \ldots\}$ were defined on the free monoid $X^{*}$ generated by the alphabet $X=\{a, b\}$. In this paper we investigate some algebraic properties of these two types of Fibonacci languages. We show that a general Fibonacci language is a homomorphical image of either $F_{a, b}^{1}$ of $F_{a, b}^{0}$. We also study the properties of Fibonacci language related to formal language theory and codes. We obtained the facts that every Fibonacci word is a primitive word and for any $u \in X^{+}, u^{4}$ is not a subword of any words in both $F_{a, b}^{1}$ and $F_{a, b}^{0}$.
\end{abstract}

\section{Introduction}

This paper is a study of some algebraic properties of Fibonacci languages. Let $X^{*}$ be the free monoid generated by an alphabet $X$ consisting of exactly two letters, i.e., $X=\{a, b\}$. Any element of $X^{*}$ is called a word over $X$ and any subset of $X^{*}$ is called a language over $X$. Let $X^{+}=X^{*} \backslash\{1\}$, where 1 is the empty word. The length of a word $u \in X^{*}$ is denoted by $l g(u)$.

Let $w_{1}=u, w_{2}=v$, where $u, v \in X^{+}$. The two types of Fibonacci sequences are defined recursively as follows:

(1) $w_{1}=u, w_{2}=v, w_{3}=u v, \ldots, w_{n}=w_{n-2} w_{n-1}, w_{n+1}=w_{n-1} w_{n}, \ldots$;

(2) $w_{1}=u, w_{2}=v, w_{3}=v u, \ldots, w_{n}=w_{n-1} w_{n-2}, w_{n+1}=w_{n} w_{n-1}, \ldots$

We note that for $n \geq 4,2 \cdot \lg \left(w_{n-2}\right)<\lg \left(w_{n}\right)<2 \cdot \lg \left(w_{n-1}\right)$.

Let $F_{u, v}^{1}$ be the set formed by taking the union of the first sequence and $F_{u, v}^{0}$ be the set formed by taking the union of the second sequence respectively. That is

$$
F_{u, v}^{1}=\{u, v, u v, v u v, u v v u v, \ldots\}
$$

and

Received April 25, 1995.

1991 Mathematics Subject Classification. 68R15, 68Q45

Key words and phrases. Fibonacci language, regular-free language, code, repetitive subword, primitive word. 


$$
F_{u, v}^{0}=\{u, v, v u, v u v, v u v v u, \ldots\}
$$

We will call $F_{u, v}^{1}$ a Fibonacci language of type 1 and $F_{u, v}^{0}$ a Fibonacci language of type 0 respectively. If we take the initial words $w_{1}$ and $w_{2}$ to be the letters $a$ and $b$, then we get the two types of Fibonacci languages

$$
F_{a, b}^{1}=\{a, b, a b, b a b, a b b a b, \ldots\} \quad \text { and } \quad F_{a, b}^{0}=\{a, b, b a, b a b, b a b b a, \ldots\}
$$

These Fibonacci languages will be the simplest ones and will be called the 1-atom Fibonacci language and 0 -atom Fibonacci language respectively. We will see that any other Fibonacci languages will related to one of these two atom Fibonacci languages. For convenience we will consider a Fibonacci language also as a sequence. In order to discuss properties on languages, we need to define some terms which we need. For any two languages $A, B \subseteq X^{*}$, let $A B=\{x y \mid x \in A, y \in B\}$ and $A^{*}=A^{0} \cup A \cup A^{2} \cup A^{3} \cup \cdots$, where $A^{0}=\{1\}$. If $u$ is a word such that $u=x w y$, where $w \in X^{+}, x, y \in X^{*}$, then the word $w$ will be called a subword of $u$. A subword $w$ of $u$ is a proper subword of $u$ if $u=x w y$ such that $x, y \in X^{+}$. For $u \in X^{+}$, let $E(u)$ and $\bar{E}(u)$ be the set of all subwords of $u$ and the set of all proper subwords of $u$ respectively. A word $u \in X^{+}$is called a primitive word if it can not be written as a power of any other word. We will let $Q$ be the set of all primitive words over $X$. And for $n \geq 2$, we let $Q^{(n)}=\left\{f^{n} \mid f \in Q\right\}$. In particular $Q^{(1)}=Q$. We call the word $u$ an overlapping word if $u$ is such that $u=w x=y w$ for some $w \in X^{+}, x, y \in X^{+}$. Let $u, v \in X^{+}$. We say that $v$ is a conjugate of $u$ if $u=x y$, for some $x, y \in X^{*}$, then $v=y x$. It is easy to see that if $v$ is a conjugate of $u$, then $u$ is a conjugate of $v$. Thus we may call two words with such a property a conjugate pair. It is known that $x y \in Q^{(n)}$ if and only if $y x \in Q^{(n)}$ for any $n \geq 1([3])$. A square free word is a word such that every subword of $u$ is primitive. A word $u$ is cubic-free if no subword of $u$ is $x^{3}$ for some $x \in X^{+}$. We let $G$ be the set of all square free words over $X$. If $X=\{a, b\}$, then $G=\{a, b, a b, b a, a b a, b a b\}$. Let $G^{(1)}=G_{1}=G$ and also for any $n \geq 2$, let the set $G_{n}=\left\{u \in X^{+} \mid E(u) \cap Q^{(n)} \neq \emptyset\right\}$ and $G^{(n)}=G_{n} \backslash G_{n+1}$. Then clearly,

$$
X^{+}=G^{(1)} \cup G^{(2)} \cup G^{(3)} \cup \cdots,
$$

and the union is a disjoint union. We note that the set $G^{(1)} \cup G^{(2)}$ consists of all cubic-free words over $X$. A language $L \subseteq X^{+}$is a code if $L$ generates a free submonoid of $X^{*}$. A 2-code $A$ is a language such that every two elements from $A$ is a code.

In this paper we investigate mostly the algebraic properties of the two types of atom Fibonacci languages. We show that every atom Fibonacci word is a primitive word. It is proved that the two types of atom Fibonacci words of the same length is a conjugate pair. Both $F_{a, b}^{1}$ and $F_{a, b}^{0}$ are 2-codes and each partitioned into two infinite subsets $F_{1}^{1}, F_{2}^{1}$ and $F_{1}^{0}, F_{2}^{0}$, all of them are codes. A language $L \subseteq X^{*}$ is regular if $L$ can be recognized by an automaton or equivalently the index of the principal congruence determined by the language $L$ is finite. We also obtained the fact that both atom Fibonacci languages are 
regular free languages in the sense that every infinite subset of it is not a regular language. There are some atom Fibonacci words which are not cubic free. We obtained that for any $u \in X^{+}, u^{4}$ is not a subword of any atom Fibonaccci words. The terminologies not defined in here please refer to [3].

\section{Elementary Properties of the Languages $F_{a, b}^{0}$ and $F_{a, b}^{1}$}

A mapping $h$ from $X^{+}$into $X^{+}$is called a homomorphism if $h(u v)=h(u) h(v)$ for all $u, v \in X^{+}$. For a given homomorphism $h: X^{+} \rightarrow X^{+}$and for any language $L \subseteq X^{+}$, we let $h(L)=\{h(u) \mid u \in L\}$. The substitution of the form, for $x, y \in X^{+}$,

$$
a \rightarrow x, b \rightarrow y
$$

induces a homomorphism from $X^{+}$into $X^{+}$. For example, if we let $h: X^{+} \rightarrow X^{+}$by $h(a)=u, h(b)=v, u, v \in X^{+}$be two fixed words, then $h$ is clearly a homomorphism. Thus the following lemma is immediate.

Lemma 2.1. Every Fibonacci sequence is a homomorphic image of the atom Fibonacci sequences $F_{a, b}^{1}$ or $F_{a, b}^{0}$.

Due to above fact, some algebraic properties of the atom Fibonacci languages can be carried through by a homomorphism. The study of the algebraic properties on the atom Fibonacci languages is then important.

In [2] de Luca has proved that every word in $F_{a, b}^{0}$ is a primitive word. In here we give a different proof to show that words in both $F_{a, b}^{1}$ and $F_{a, b}^{0}$ are primitive words. In order to do this we need the following known result. Recall that a non-empty word $f$ is a primitive word if $f$ is not a power of any other word.

Lemma 2.2. ([4], [5]) Let $a, c$ be two primitive words. If $a^{m}=c^{k} x\left(a^{m}=x c^{k}\right)$, $m, k \geq 2$ and $x$ is a prefix (suffix) of $c$, then $a=c$.

Proposition 2.3. The atom Fibonacci languages $F_{a, b}^{0}$ and $F_{a, b}^{1}$ are subsets of $Q$.

Proof. Consider $F_{a, b}^{0}=\left\{a, b, b a, b a b, b a b b a, b a b b a b a b, \cdots, w_{n}, w_{n+1}, w_{n+2}, \cdots\right\}$. We show that every word in $F_{a, b}^{0}$ is a primitive word by using the above lemma. The proof will be completed by induction on terms. We observe that the first few terms in $F_{a, b}^{0}$ are primitive words. Now we assume that $w_{1}, w_{2}, \ldots, w_{n-1}, w_{n}$ are primitive words and we show that, for $n \geq 3$, the term $w_{n+1}$ is also a primitive word. We note that $w_{n}=$ $w_{n-1} w_{n-2}$ and

$$
w_{n+1}=w_{n-1} w_{n-2} w_{n-1}
$$

Since $x y$ is a primitive word if and only if $y x$ is a primitive word, (Proposition 1.11 [3]), to show that $w_{n+1}$ is a primitive word is equivalent to show that the word

$$
u=\left(w_{n-1}\right)^{2} w_{n-2}
$$


is primitive. Suppose the word $u=f^{m}, m \geq 2, f$ is a primitive word. Since $w_{n-2}$ is a prefix of $w_{n-1}$ in our sequence, by the above lemma, we see that $f=w_{n-1}$, which is not possible. Thus the word $u$ is primitive. Therefore $w_{n+1}$ is a primitive word. By induction conclusion, we have that every Fibonacci word in $F_{a, b}^{0}$ is a primitive word.

For the case of $F_{a, b}^{1}$, since each Fibonacci word $w_{n}$ is a suffix of $w_{n+1}$, by a similar argument and with a help of the above lemma, we can prove that every Fibonacci word in $F_{a, b}^{1}$ is a primitive word.

Proposition 4.9 in section 4 generalized the present result.

\section{Conjugate Property of two Types of Fibonacci Words}

In the two types of atom Fibonacci languages $F_{a, b}^{0}$ and $F_{a, b}^{1}$, the corresponding terms of Fibonacci words are of the same length. In the following, we show that they are in fact a conjugate pair. For convenience, we use the notation $u \leq_{p} v$ to mean that the word $u$ is a prefix of the word $v$ and $u \leq_{s} v$ to mean that the word $u$ is a suffix of the word $v$.

To prove the following, we let

$$
\begin{gathered}
F_{a, b}^{0}=\{a, b, b a, b a b, b a b b a, \ldots\}=\left\{z_{1}, z_{2}, z_{3}, z_{4}, z_{5}, \ldots\right\} \\
F_{a, b}^{1}=\{a, b, a b, b a b, a b b a b, \ldots\}=\left\{w_{1}, w_{2}, w_{3}, w_{4}, w_{5}, \ldots\right\} .
\end{gathered}
$$

Lemma 3.1. For $n \geq 1$, let $z_{n}, w_{n}$ be the $n$-th words in $F_{a, b}^{0}$ and $F_{a, b}^{1}$ respectively. For $n \geq 3$, let $z_{n}=z_{n}^{\prime} z_{n}^{\prime \prime}, w_{n}=w_{n}^{\prime} w_{n}^{\prime \prime}$, where $z_{n}^{\prime}, z_{n}^{\prime \prime}, w_{n}^{\prime}, w_{n}^{\prime \prime} \in X^{*}$ with $\lg \left(z_{n}^{\prime \prime}\right)=\lg \left(w_{n}^{\prime}\right)=2$. Then

$$
z_{n}^{\prime}=w_{n}^{\prime \prime}
$$

Proof. First, by observation for the $n$-th terms $z_{n}$ in $F_{a, b}^{0}$ and $w_{n}$ in $F_{a, b}^{1}$, we have that $\lg \left(z_{n}\right)=\lg \left(w_{n}\right)$ and $z_{3}=b a, \quad z_{4}=b a b, \quad w_{3}=a b, \quad w_{4}=b a b$. Clearly, $b a \leq_{s} z_{3}, \quad a b \leq_{s} z_{4}, \quad a b \leq_{p} w_{3}, \quad b a \leq_{p} w_{4}$. Since $z_{n+2}=z_{n+1} z_{n} ; \quad w_{n+2}=w_{n} w_{n+1}$, clearly for $n \geq 3$, we have that, for some $z_{n}^{\prime}, w_{n}^{\prime \prime} \in X^{*}$,

$$
\begin{gathered}
z_{n}= \begin{cases}z_{n}^{\prime} b a, & \text { if } \mathrm{n} \text { is odd } ; \\
z_{n}^{\prime} a b, & \text { if } \mathrm{n} \text { is even; }\end{cases} \\
w_{n}= \begin{cases}a b w_{n}^{\prime \prime}, & \text { if } \mathrm{n} \text { is odd } ; \\
b a w_{n}^{\prime \prime}, & \text { if } \mathrm{n} \text { is even. }\end{cases}
\end{gathered}
$$

Our proof will be completed by induction on terms $n \geq 3$.

(1) It is clear that for $n=3, z_{3}=b a=(1)(b a), w_{3}=a b=(a b)(1), z_{3}^{\prime}=1=w_{3}^{\prime \prime}$; for $n=4, z_{4}=(b)(a b), w_{4}=(b a)(b), z_{4}^{\prime}=b=w_{4}^{\prime \prime}$. Thus the lemma is true for $n=3$ and 4 . 
(2) Suppose $z_{n}^{\prime}=w_{n}^{\prime \prime}$, for $3 \leq n \leq m$.

(3) Let $n=m+1$. We consider the following two cases:

(a) If $m$ is odd, then $n=m+1$ is even, by Equations (3.3) and (3.4) and by induction hypothesis,

$$
\begin{aligned}
z_{n}=z_{m+1} & =z_{m-1} z_{m} \\
& =z_{m-1} z_{m-2} z_{m-1} \\
& =z_{m-1}^{\prime} a b z_{m-2}^{\prime} b a z_{m-1}^{\prime} a b \\
& =w_{m-1}^{\prime \prime} a b w_{m-2}^{\prime \prime} b a w_{m-1}^{\prime \prime} a b \\
& =w_{m-1}^{\prime \prime} w_{m-2} w_{m-1} a b \\
& =w_{m-1}^{\prime \prime} w_{m} a b .
\end{aligned}
$$

On the other hand, by Equation (3.3), $z_{m+1}=z_{m+1}^{\prime} a b$, we have then $z_{m+1}^{\prime}=$ $w_{m-1}^{\prime \prime} w_{m}$. That is,

$$
z_{n}^{\prime}=w_{n-2}^{\prime \prime} w_{n-1} \text {. }
$$

Similarly, by Equation (3.4),

$$
\begin{aligned}
w_{m+1} & =w_{m-1} w_{m} \\
& =b a w_{m-1}^{\prime \prime} w_{m} .
\end{aligned}
$$

Again by Equation (3.4), $w_{m+1}=b a w_{m+1}^{\prime \prime}$, then we have that $w_{m+1}^{\prime \prime}=w_{m-1}^{\prime \prime} w_{m}$. That is,

$$
w_{n}^{\prime \prime}=w_{n-2}^{\prime \prime} w_{n-1} .
$$

Now by Equations (3.5) and (3.6), $z_{n}^{\prime}=w_{n}^{\prime \prime}$.

(b) If $m$ is even, then $m+1$ is odd. By a similar argument as in the case (a), we can show that $z_{n}^{\prime}=w_{n}^{\prime \prime}$.

Summing up Cases (a), (b) and by induction conclusion, we have that $z_{n}^{\prime}=w_{n}^{\prime \prime}$, for $n \geq 3$.

Lemma 3.2. For $n \geq 3$, let $z_{n}=z_{n}^{\prime} z_{n}^{\prime \prime}, w_{n}=w_{n}^{\prime} w_{n}^{\prime \prime}$, where $z_{n}, w_{n}, z_{n}^{\prime}, z_{n}^{\prime \prime}, w_{n}^{\prime}, w_{n}^{\prime \prime}$ are the same definitions as in Lemma 3.1. Then

$$
z_{n+1}^{\prime \prime} z_{n}=w_{n} w_{n+1}^{\prime}
$$

Proof. First, by Equations (3.3) and (3.4), we have

$$
\begin{aligned}
& z_{n}^{\prime \prime}= \begin{cases}b a, & \text { if } \mathrm{n} \text { is odd } \\
a b, & \text { if } \mathrm{n} \text { is even }\end{cases} \\
& w_{n}^{\prime}= \begin{cases}a b, & \text { if } \mathrm{n} \text { is odd } \\
b a, & \text { if } \mathrm{n} \text { is even }\end{cases}
\end{aligned}
$$


Now we consider following two cases:

(1) If $n=2 k+1, k \geq 1$, then, by Lemma 3.1 and Equations (3.3), (3.4), (3.7), (3.8),

$$
\begin{aligned}
z_{n+1}^{\prime \prime} z_{n} & =a b z_{2 k+1} \\
& =a b z_{2 k+1}^{\prime} b a \\
& =a b w_{2 k+1}^{\prime \prime} b a \\
& =w_{2 k+1} b a \\
& =w_{n} w_{n+1}^{\prime} .
\end{aligned}
$$

(2) If $n=2 k+2, k \geq 1$, then, by the similar argument as in (1), we have that $z_{n+1}^{\prime \prime} z_{n}=$ $w_{n} w_{n+1}^{\prime}$.

By Cases (1) and (2), then for all $n \geq 3, z_{n+1}^{\prime \prime} z_{n}=w_{n} w_{n+1}^{\prime}$.

Proposition 3.3. Two Fibonacci words in $F_{a, b}^{0}$ and $F_{a, b}^{1}$ of the same length form a conjugate pair.

Proof. Consider Equations (3.1) and (3.2). By observation, we have that $z_{1}=$ $w_{1}, z_{2}=w_{2}, z_{3}=b a, w_{3}=a b$ and $z_{4}=w_{4}$. Thus, for $n \leq 4, z_{n}$ and $w_{n}$ form a conjugate pair.

We now show that for $n \geq 5, z_{n}$ and $w_{n}$ form a conjugate pair. By Lemmas 3.1 and 3.2 , we have that, for $n \geq 3$,

$$
\begin{aligned}
z_{n+2} & =z_{n+1} z_{n} \\
& =z_{n+1}^{\prime} z_{n+1}^{\prime \prime} z_{n} \\
& =w_{n+1}^{\prime \prime} w_{n} w_{n+1}^{\prime}
\end{aligned}
$$

and

$$
\begin{aligned}
w_{n+2} & =w_{n} w_{n+1} \\
& =w_{n} w_{n+1}^{\prime} w_{n+1}^{\prime \prime} .
\end{aligned}
$$

It is now clear that $z_{n+2}$ and $w_{n+2}$ form a conjugate pair. We then conclude that for $n \geq 5, z_{n}$ and $w_{n}$ form a conjugate pair.

\section{Fibonacci Languages Related to Codes and Formal Language The- ory}

From Lemma 1.7 ([4]) and Proposition 1.25 ([4]), we have that every non-empty subset of $Q$ is a 2-code. The following lemma follows from Proposition 2.3.

Lemma 4.1. The atom Fibonacci languages $F_{a, b}^{0}$ and $F_{a, b}^{1}$ are 2-codes.

Lemma 4.2. (1) For Fibonacci words $w_{n}, w_{n+2}, w_{n+3}$ in $F_{a, b}^{1}$, we have $w_{n+2}$ $w_{n+2}=w_{n} w_{n+3}$. (2) For Fibonacci words $z_{n}, z_{n+2}, z_{n+3}$ in $F_{a, b}^{0}$, we have $z_{n+2} z_{n+2}=$ $z_{n+3} z_{n}$. 
Proof. (1) Since $w_{n+3}=w_{n+1} w_{n+2}$, we have

$$
\begin{aligned}
w_{n} w_{n+3} & =w_{n}\left(w_{n+1} w_{n+2}\right) \\
& =\left(w_{n} w_{n+1}\right) w_{n+2} \\
& =w_{n+2} w_{n+2} .
\end{aligned}
$$

(2) By a similar way, we can show that (2) holds true.

From the sequences $F_{a, b}^{0}$ and $F_{a, b}^{1}$, we see that for any $n \geq 1$, the set $\left\{w_{n}, w_{n+1}, w_{n+2}\right\}$ is not a code. From the above lemma, we see that, for $n \geq 1$, the set $\left\{w_{n}, w_{n+2}, w_{n+3}\right\}$ is not a code. Moreover, for any code $A \subset F_{a, b}^{1}$ such that $w_{m}, w_{m+1} \in A$, for some $m \geq 1$, then, clearly, $w_{m+i} \notin A$, for all $i \geq 2$. Thus if a subset $A$ of $F_{a, b}^{1}$ is a code and contains two consecutive Fibonacci words, then the set $A$ must be finite. Nevertheless, we will show that $F_{a, b}^{1}$ and $F_{a, b}^{0}$ contain infinite subsets which are codes. For constructing such infinite subsets, we just do by partitioning the two types of atom Fibonacci languages. First we need the following

Proposition 4.3. ([4]) Let $A \subseteq X^{+}$. Then $A$ is a code if and only if $x_{1} x_{2} \cdots x_{n}=y_{1} y_{2} \cdots y_{n}, x_{i}, y_{i} \in A, i=1,2, \ldots, n$ implies $x_{i}=y_{i}, i=1,2, \ldots, n$.

Let

$$
\begin{gathered}
F_{a, b}^{0}=\{a, b, b a, b a b, b a b b a, \ldots\}=\left\{z_{1}, z_{2}, z_{3}, z_{4}, z_{5}, \ldots\right\} \\
F_{a, b}^{1}=\{a, b, a b, b a b, a b b a b, \ldots\}=\left\{w_{1}, w_{2}, w_{3}, w_{4}, w_{5}, \ldots\right\}
\end{gathered}
$$

and let

$$
\begin{aligned}
& F_{1}^{1}=\left\{w_{n} \in F_{a, b}^{1} \mid n=1,3,5,7, \ldots\right\} \\
&=\left\{w_{1}, w_{3}, w_{5}, w_{7}, \ldots\right\} \\
&=\{a, a b, a b b a b, a b b a b b a b a b b a b, \ldots\} ; \\
& F_{2}^{1}=\left\{w_{n} \in F_{a, b}^{1} \mid n=2,4,6,8, \ldots\right\} \\
&=\left\{w_{2}, w_{4}, w_{6}, w_{8}, \ldots\right\} \\
&=\{b, b a b, b a b a b b a b, b a b a b b a b a b b a b b a b a b b a b, \ldots\} ; \\
& F_{1}^{0}=\left\{z_{n} \in F_{a, b}^{0} \mid n=1,3,5,7, \ldots\right\} \\
&=\left\{z_{1}, z_{3}, z_{5}, z_{7}, \ldots\right\} \\
&=\{a, b a, b a b b a, b a b b a b a b b a b b a, \ldots\} \\
& F_{2}^{0}=\left\{z_{n} \in F_{a, b}^{0} \mid n=2,4,6,8, \ldots\right\} \\
&=\left\{z_{2}, z_{4}, z_{6}, z_{8}, \ldots\right\} \\
&=\{b, b a b, b a b b a b a b, b a b b a b a b b a b b a b a b b a b a b, \ldots\} .
\end{aligned}
$$

At this stage we like to give the following remarks: 


\section{Remark.}

(1) No words in $F_{1}^{1}$ is a prefix of any words in $F_{2}^{1}$ and also no words in $F_{2}^{1}$ is a prefix of any words in $F_{1}^{1}$.

(2) Every $n$-th word in $F_{1}^{1}$ is a prefix and also a suffix of $(n+1)$-th word in $F_{1}^{1}, n \geq 2$.

(3) Every $n$-th word in $F_{2}^{1}$ is a prefix and also a suffix of $(n+1)$-th word in $F_{2}^{1}, n \geq 1$.

We claim that the four subsets $F_{1}^{1}, F_{2}^{1}, F_{1}^{0}$ and $F_{2}^{0}$ are codes. First, we prove the following some lemmas.

Lemma 4.4. Let $F_{1}^{1}$ be defined by Equation (4.3). If $w_{n} \in F_{1}^{1}, n \geq 1$, and $w_{n}=x_{1} x_{2}$, for $x_{1} \in F_{1}^{1}$ and $x_{2} \in X^{+}$, then $b \leq_{p} x_{2}$.

Proof. Let $w_{n} \in F_{1}^{1}$. Then $n$ is odd. We have

$$
\begin{aligned}
w_{n} & =\left(w_{n-2}\right) w_{n-1} \\
& =\left(w_{n-4}\right) w_{n-3} w_{n-1} \\
& =\left(w_{n-6}\right) w_{n-5} w_{n-3} w_{n-1} \\
& =\cdots \\
& =\left(w_{1}\right) w_{2} w_{4} \cdots w_{n-1} .
\end{aligned}
$$

In each expression above for $w_{n}$, we see that

$$
w_{n-2}, w_{n-4}, w_{n-6}, \cdots, w_{1}
$$

are in $F_{1}^{1}$, and $w_{2}, w_{4}, \ldots, w_{n-3}, w_{n-1}$ are in $F_{2}^{1}$. It is now clear that $b \leq_{p} x_{2}$.

The following is a dual case of the above lemma.

Lemma 4.5. Let $F_{2}^{1}$ be defined by Equation (4.4). If $w_{n} \in F_{2}^{1}, n \geq 1$ and $w_{n}=x_{1} x_{2}$, for $x_{1} \in F_{2}^{1}, x_{2} \in X^{+}$, then $a \leq_{p} x_{2}$.

Corollary 4.6. Let $F_{1}^{0}, F_{2}^{0}$ be defined by Equation (4.5), (4.6), respectively If $z_{n}, n \geq 1$, is in the set $F_{1}^{0}\left(F_{2}^{0}\right)$ and $z_{n}=x_{1} x_{2}$, for $x_{1} \in F_{1}^{0}\left(F_{2}^{0}\right), x_{2} \in X^{+}$, then $x_{1}, x_{2}$ do not have the same suffixes.

Proposition 4.7. The languages $F_{1}^{1}, F_{2}^{1}, F_{1}^{0}$ and $F_{2}^{0}$ are codes.

Proof. We only prove that $F_{1}^{1}$ is a code and the proofs of the rest are similar.

By Proposition 4.3, we consider $x_{1} x_{2} \cdots x_{n}=y_{1} y_{2} \cdots y_{n}$, for $n \geq 1$ and $x_{1}, x_{2}, \ldots, x_{n}$, $y_{1}, y_{2}, \ldots, y_{n} \in F_{1}^{1}$. We will show that $x_{i}=y_{i}, i=1,2, \ldots, n$. Our proof will be completed by induction on $n$. The proposition holds true for the case $n=1$ and we assume that the proposition is true for all $n<k$.

Now let $n=k$ and suppose $x_{1} x_{2} \cdots x_{n}=y_{1} y_{2} \cdots y_{n}$. First, we show that $x_{1}=y_{1}$. Suppose on the contrary $x_{1} \neq y_{1}$ and no loss of generality, we assume that $\lg \left(x_{1}\right) \geq \lg \left(y_{1}\right)$. (i) If $l g\left(x_{1}\right)=l g\left(y_{1}\right)$, then $x_{1}=y_{1}$. (ii) If $l g\left(x_{1}\right)>l g\left(y_{1}\right)$, then we let $x_{1}=y_{1} y_{2} \cdots y_{i-1} y_{i}^{\prime}$, 
for some $1 \leq i<n, y_{i}^{\prime} \in X^{+}, y_{i}=y_{i}^{\prime} y_{i}^{\prime \prime}, y_{i}^{\prime \prime} \in X^{*}$. If $i \geq 2$, then since $x_{1}, y_{1} \in F_{1}^{1}$, by Lemma $4.4, b \leq_{p} y_{2}$. While since $y_{2} \in F_{1}^{1}, a \leq_{p} y_{2}$, a contradiction. Hence $x_{1}=y_{1}$. Thus, by both Cases (i) and (ii), $x_{1}=y_{1}$ and $x_{2} x_{3} \cdots x_{n}=y_{2} y_{3} \cdots y_{n}$. Now by induction hypothesis, we have $x_{i}=y_{i}, i=2,3, \ldots, n$. Hence $x_{i}=y_{i}, i=1,2,3, \ldots, n$. By induction conclusion, we have that $F_{1}^{1}$ is a code.

We are now in the position to discuss some language properties of the homomorphical image of the atom Fibonacci language $F_{a, b}^{0}$ by using the following known result. A code $L \subseteq X^{+}$is a pure code means that for any $x \in L^{*}$, the primitive root of $x, \sqrt{x}$ is in $L^{*}$ (see [4]).

Proposition 4.8. ([4]) Let $h: X^{*} \rightarrow X^{*}$ be an injective homomorphism. If $h(X)$ is a pure code, then $h$ preserves the primitive words.

Proposition 4.9. Let $h: X^{+} \rightarrow X^{+}$be an injective homomorphism such that $h(a)=u, h(b)=v$, where $a, b \in X$. Then the Fibonaccci language $F_{u, v}^{1}=h\left(F_{a, b}^{1}\right)$ is a subset of $Q$ if $\{u, v\}$ is a pure code.

Proof. A direct consequence of the above proposition.

The set $\{u, v\}$ in the above proposition will be called the core of the Fibonacci language $F_{u, v}^{1}$. In the following, we consider the Fibonacci languages which are homomorphic images of $F_{a, b}^{1}$ in which the set $\{h(a)=u, h(b)=v\}$ is not a code.

Proposition 4.10. Let $F_{u, v}^{1}$ be a Fibonacci language such that $h(a)=$ $u, h(b)=v$. Then $F_{u, v}^{1} \subseteq f^{+}$, for some primitive word $f$, if and only if $\{u, v\}$ is not a code.

Proof. $(\Rightarrow)$ Suppose for some primitive word $f$ such that $F_{u, v}^{1} \subseteq f^{+}$. Then clearly, $u, v \in f^{+}$and $\{u, v\}$ is not a code.

$(\Leftarrow)$ Assuming that $\{u, v\}$ is not a code. Then by Lemma 1.25 of [4], uv $=v u$. By Lemma 1.7 of [4] the words $u$ and $v$ are powers of a common primitive word. Let the primitive word be $f$. Then clearly the Fibonacci language $F_{u, v}^{1}$ is a subset of $f^{+}$.

Remark. Every atom Fibonacci word is an overlapping word except for the first three terms.

Proposition 4.11. ([4]) Let $h: X^{*} \rightarrow X^{*}$ be a homomorphism. Then the following are equivalent:

(1) $h$ is injective;

(2) $h(X)$ is a code and $|h(X)|=|X|$;

(3) $h$ preserves codes;

(4) $h$ preserves 2 -codes.

We note that two-element set is a 2-code if and only if it is a code. 
Proposition 4.12. The Fibonacci core $\{u, v\}, u \neq v$, is a code if and only if the Fibonacci language $F_{u, v}^{1}$ is a 2-code.

Proof. $(\Rightarrow)$ The atom Fibonacci language $F_{a, b}^{1}$ is a 2-code, and $F_{u, v}^{1}$ is an injective homomorphical image of $F_{a, b}^{1}$, by the above proposition, the conclusion holds.

$(\Leftarrow)$ Since $F_{u, v}^{1}$ is a 2-code, the first two Fibonacci word $\{u, v\}$ forms a 2-code and hence $\{u, v\}$ is a code.

We remark here that if $\{u, v\}$ is a code, $h$ an injective homomorphism and $h(a)=$ $u, h(b)=v$, then the homomorphical images $h\left(F_{1}^{1}\right)$ and $h\left(F_{2}^{1}\right)$ of $F_{1}^{1}$ and $F_{2}^{1}$, respectively, are codes. That is both the sets $\left\{h\left(w_{n}\right) \mid n=2,4,6,8, \ldots\right\}$ and $\left\{h\left(w_{n}\right) \mid n=1,3,5,7, \ldots\right\}$ are codes.

In the following we consider the atom Fibonacci languages related to formal language theory. We will see that both the two languages $F_{a, b}^{0}$ and $F_{a, b}^{1}$ contain no infinite regular subsets. In other words, both $F_{a, b}^{0}$ and $F_{a, b}^{1}$ are regular free.

For a given language $L \subseteq X^{*}$, the principal congruence $P_{L}$ determined by $L$ is defined as follows: for any $u, v \in X^{*}$,

$$
u \equiv v\left(P_{L}\right) \Longleftrightarrow\left(x u y \in L \Longleftrightarrow x v y \in L \text { for all } x, y \in X^{*}\right) .
$$

In formal language theory, we call the language $L \subseteq X^{*}$ regular if the index of $P_{L}$ is finite. An infinite language $L$ is a regular free language if any infinite subset of $L$ is not a regular language. We now show the following:

Proposition 4.13. The language $F_{a, b}^{1}\left(F_{a, b}^{0}\right)$ is regular free.

Proof. We show that $F_{a, b}^{1}$ contains no infinite regular subsets and the proof of the other case is similar. Let $A=\left\{u_{1}, u_{2}, u_{3}, \ldots\right\} \subseteq\left\{w_{1}, w_{2}, w_{3}, \ldots\right\}$ be an infinite subset of $F_{a, b}^{1}$. We show that for any $u_{i}, u_{j} \in A, i<j$,

$$
u_{i} \not \equiv u_{j}\left(P_{A}\right) \text {. }
$$

This is true, for if $u_{i}=w_{r}, u_{j}=w_{s}$, then $r \neq s$. Let $x, y \in X^{*}$ be such that $x u_{i} y=x w_{r} y \in$ $A$ with the length of $x y$ is minimal. Then by the fact that the length of Fibonacci words are Fibonacci numbers, clearly the word $x u_{j} y$ is not in $A$. This shows that $u_{i} \not \equiv u_{j}\left(P_{A}\right)$ if $i \neq j$. It follows that the index of $P_{A}$ is infinite and $A$ is not regular.

\section{The Problem of Repetitive Subwords in $F_{a, b}^{1}$}

Lemma 5.1 Let $\{u, v\} \subset Q$ be a conjugate pair and let $u=x y, v=y x$, for some $x, y \in X^{+}$. Then $u \neq v$.

Proof. Suppose on the contrary $u=v$. Then $x y=y x$, for $x, y \in X^{+}$. By Lemma $1.7([4])$, we have that $x$ and $y$ are powers of a common word. It follows that $u, v \notin Q$, a contradiction. Thus $u \neq v$ must be true. 
Proposition 5.2. For $n \geq 1$, let $z_{n}, w_{n}$ be the $n$-th words in $F_{a, b}^{0}$ and $F_{a, b}^{1}$ respectively. Then $z_{n} \neq w_{n}$, except $n=1,2,4$.

Proof. The situation is clear for the first five terms. By Proposition 3.3, for $n \geq 6$, $\left\{z_{n}, w_{n}\right\}$ is a conjugate pair in the forms:

$$
z_{n}=w_{n-1}^{\prime \prime}\left(w_{n-2} w_{n-1}^{\prime}\right)
$$

and

$$
w_{n}=\left(w_{n-2} w_{n-1}^{\prime}\right) w_{n-1}^{\prime \prime} .
$$

Since $w_{n-1}^{\prime \prime}, w_{n-2} w_{n-1}^{\prime} \in X^{+}$and $z_{n}, w_{n} \in Q$, by Lemma 5.1, we have that $z_{n} \neq w_{n}$.

Lemma 5.3. Let $F_{1}^{1}, F_{2}^{1}$ be the codes defined in Equations (4.3), (4.4) respectively. Let $w \in F_{a, b}^{1}$. The following are true:

(1) If $w \in F_{1}^{1}$ and $u \in F_{1}^{1}$ is a prefix of $w$, then $w=u v x$ for some $v \in F_{2}^{1}$ and $x \in X^{*}$.

(2) If $w \in F_{2}^{1}$ and $u \in F_{2}^{1}$ is a prefix of $w$, then $w=u v x$ for some $v \in F_{1}^{1}$ and $x \in X^{*}$.

Proof. The result is clear from the proof of Lemmas 4.4 and 4.5 .

Lemma 5.4. Let $w_{n} \in F_{a, b}^{1}, n \geq 1$. Then, for the Fibonacci word $w_{i}$, the following are true: (1) $\left(w_{i}\right)^{2} \underline{\Sigma}_{p} w_{n}$, for $i<n$; (2) $w_{i} \leq_{s} w_{n}$, for $n \geq 3$ and $2 \leq i \leq n-1 ;(3)\left(w_{i}\right)^{2} \leq_{s} w_{n}$, for $n \geq 6$ and $4 \leq i \leq n-2 ;(4)\left(w_{i}\right)^{3} \mathbb{L}_{s} w_{n}$, for $i<n$.

Proof. (1) Since the words in $F_{1}^{1}$ start with the letter $a$ and the words in $F_{2}^{1}$ start with the letter $b$, the result holds from Lemmas 4.4 and 4.5 .

(2) Since $w_{n}=w_{n-2} w_{n-1}=w_{n-2} w_{n-3} w_{n-2}=\cdots$ and by the recursion of the words in $F_{a, b}^{1}$, we conclude that the result is true.

(3) Consider $w_{k}=w_{k-2} w_{k-1}, k>3$ and from Lemma $4.2(1), w_{k-1} w_{k-1}=w_{k-3} w_{k}$, we have

$$
\begin{aligned}
w_{k} w_{k-1} & =w_{k-2} w_{k-1} w_{k-1} \\
& =w_{k-2} w_{k-3} w_{k} .
\end{aligned}
$$

By applying the Equation (5.1), we have $w_{n-2} w_{n-3}=w_{n-4} w_{n-5} w_{n-2}$ and

$$
\begin{aligned}
w_{n} & =w_{n-2} w_{n-1} \\
& =w_{n-2} w_{n-3} w_{n-2} \\
& =w_{n-4} w_{n-5} w_{n-2} w_{n-2} .
\end{aligned}
$$

We now show that $\left(w_{i}\right)^{2} \leq_{s} w_{n}$, for $n \geq 6$ and $4 \leq i \leq n-2$. We do this by induction on $n$.

(i) For $n=6, w_{6}=b a b a b b a b$, since $\left(w_{4}\right)^{2} \leq_{s} w_{6}$ and $n-2=4$, the result is true. 
(ii) Suppose for some $k \geq 6$, and for every $6 \leq n \leq k$, such that $\left(w_{i}\right)^{2} \leq_{s} w_{n}, 4 \leq i \leq$ $n-2$.

For $n=k+1$, by definition, $w_{n}=w_{k-1} w_{k}$. By assumption $\left(w_{i}\right)^{2} \leq_{s} w_{k}, 4 \leq i \leq k-2$ and then $\left(w_{i}\right)^{2} \leq_{s} w_{n}$. But by Equation (5.2), then .

$$
w_{k+1}=w_{k-3} w_{k-4} w_{k-1} w_{k-1}
$$

and then $\left(w_{n-2}\right)^{2}=\left(w_{k-1}\right)^{2} \leq_{s} w_{n}$. By induction conclusion, we have that for every $n \geq 6,\left(w_{i}\right)^{2} \leq_{s} w_{n}, 4 \leq i \leq n-2$.

(4) First, we observie that for the case $n \leq 5, i<n$ and for $n \geq 6$, along with the condition $i=n-1$, we have $\left(w_{i}\right)^{3} \not_{s} w_{n}$.

We now show that $\left(w_{i}\right)^{3} \underline{L}_{s} w_{n}$, for $n \geq 6$ and $i \leq n-2$. We do this by induction on $n$.

(i) For $n=6, w_{6}=b a b a b b a b$ and by observation $\left(w_{1}\right)^{3},\left(w_{2}\right)^{3},\left(w_{3}\right)^{3}$ and $\left(w_{4}\right)^{3}$ are not suffix of $w_{6}$. For $n=7, w_{7}=a b b a b b a b a b b a b$. Again by observation $\left(w_{1}\right)^{3},\left(w_{2}\right)^{3},\left(w_{3}\right)^{3}$, $\left(w_{4}\right)^{3}$ and $\left(w_{5}\right)^{3}$ are not suffix of $w_{7}$.

(ii) Suppose for some $k \geq 6$ and for every $6 \leq n \leq k,\left(w_{i}\right)^{3} \mathbb{z}_{s} w_{n}, i \leq n-2$.

Consider $n=k+1$ and $w_{n}=w_{k+1}=w_{k-1} w_{k}$. By assumption, if $i \leq n-3=k-2$, then $\left(w_{i}\right)^{3} \underline{x}_{s} w_{k} \leq_{s} w_{k+1}$. Now consider $i=n-2=k-1$, applying the Equation (5.2), we have that

$$
\begin{aligned}
w_{n} & =w_{k+1} \\
& =w_{k-3} w_{k-4} w_{k-1} w_{k-1}
\end{aligned}
$$

Since $l g\left(w_{k-3} w_{k-4}\right)=\lg \left(w_{k-4} w_{k-3}\right)=\lg \left(w_{k-2}\right)<l g\left(w_{k-1}\right)$, then we have $\left(w_{k-1}\right)^{3} \mathbb{L}_{s}$ $w_{k+1}$. Thus by induction conclusion, we have $\left(w_{i}\right)^{3} \underline{L}_{s} w_{n}, i \leq n-2$.

Lemma 5.5. Let $w_{n} \in F_{a, b}^{1}, n \geq 6$. Then $\left(w_{n-4} w_{n-5}\right)^{2} w_{n-4} \leq_{p} w_{n}$ and $\left(w_{n-4} w_{n-5}\right)^{3} \not_{p} w_{n}$.

Proof. Consider $w_{n}=w_{n-2} w_{n-1}$. Then

$$
\begin{aligned}
w_{n} & =w_{n-2} w_{n-1} \\
& =w_{n-4} w_{n-3} w_{n-3} w_{n-2} \\
& =w_{n-4} w_{n-5} w_{n-4} w_{n-5} w_{n-4} w_{n-2}
\end{aligned}
$$

Thus $\left(w_{n-4} w_{n-5}\right)^{2} w_{n-4} \leq_{p} w_{n}$. Now by the properties of $F_{1}^{1}$ and $F_{2}^{1}$, we have $w_{n-5} \mathbb{Z}_{p}$ $w_{n-2}$. It follows that $\left(w_{n-4} w_{n-5}\right)^{3} \not_{p} w_{n}$.

Proposition 5.6. Let $w_{n} \in F_{a, b}^{1}, n \geq 1$ and let $v \leq_{s} w_{n}$. If $v \leq_{p} w_{k}$, for some $w_{k} \in F_{a, b}^{1}, k \geq 1$, then $v \in F_{a, b}^{1}$.

Proof. We prove the proposition by induction on the term $n$.

(1) By observation, the proposition is true for the first 5 terms.

(2) Assume the proposition is true for the first $n$ terms, $n \geq 1$. 
Consider $w_{n+1}=w_{n-1} w_{n}$. Let $v \leq_{s} w_{n+1}$ and $v \leq_{p} w_{k}$, for some $w_{k} \in F_{a, b}^{1}$. If $\lg (v) \leq$ $l g\left(w_{n}\right)$, then $v \leq_{s} w_{n}$ and, by assumption, $v \in F_{a, b}^{1}$. If $l g(v)>l g\left(w_{n}\right)$ and let $v=$ $u w_{n}, u \in X^{+}$. Then $u \leq_{s} w_{n-1}$. From $v \leq_{p} w_{k}$, we have $u \leq_{p} v \leq_{p} w_{k}$. By induction hypothesis, $u \in F_{a, b}^{1}$. Thus $u=w_{i}$ for some $i \leq n-1$. We claim that $i=n-1$. Indeed, if $i \neq n-1$, then there exists an integer $j \geq 2$ such that $i=n-j$.

(2-1) If $j$ is even, then $u=w_{n-j} \leq_{p} w_{n}$ and then $\left(w_{n-j}\right)^{2} \leq_{p} v \leq_{p} w_{k}$. This contradicts to Lemma $5.4(1)$.

(2-2) If $j$ is odd, then, by Lemma $5.3, w_{n-j+3} \leq_{p} w_{n}$. But, by Lemma 4.2(1), $w_{n-j+2} w_{n-j+2}=w_{n-j} w_{n-j+3}$ and $\left(w_{n-j+2}\right)^{2}=w_{n-j} w_{n-j+3}=u w_{n-j+3} \leq_{p}$ $v \leq_{p} w_{k} \in F_{a, b}^{1}$

Again this contradicts to Lemma 5.4(1). We then have $v=u w_{n}=w_{n-1} w_{n}=w_{n+1} \in$ $F_{a, b}^{1}$. This completes our induction proof and the proposition holds.

Proposition 5.7. Let $w_{n}$ be the $n$-th term of the Fibonacci language $F_{a, b}^{1}$, $n \geq 1$ and let

$$
w_{n+2}=w_{n} w_{n+1}=x\left(w_{i}\right)^{3} y
$$

where $w_{i} \in F_{a, b}^{1}, i \geq 1$ and $x \leq_{p} w_{n}, y \leq_{s} w_{n+1}$. Suppose $w_{i}$ satisfies Equation (5.3) with $l g\left(w_{i}\right) \geq \lg \left(w_{j}\right)$, for every $j$ satisfies Equation (5.3). Then the following are true:

(1) If $w_{i} \leq_{p} w_{n+1}$, then $i=n-3$. That $i s, w_{n-3} \leq_{p} w_{n+1}$ and $\left(w_{n-3}\right)^{2} \leq_{s} w_{n}$.

(2) If $w_{i} \underline{\Delta}_{p} w_{n+1}$, then $i=n-2$. That is, $w_{n-4} \leq_{s} w_{n}$ and $w_{n-3}\left(w_{n-2}\right)^{2} \leq_{p} w_{n+1}$.

Proof. (1) By observation, for $n<6$, we have that $w_{n+2} \notin G_{3}$. Consider $w_{n+1}=$ $w_{n-1} w_{n}$, for $n \geq 6$. Suppose $w_{i} \leq_{p} w_{n+1}$. Then by the properties of words in $F_{a, b}^{1}$, $w_{n-k} \leq_{p} w_{n+1}$, for $k \geq 1$ and $k$ is odd. From the fact that $l g\left(\left(w_{n-1}\right)^{2}\right)>\lg \left(w_{n}\right)$, then $\left(w_{n-1}\right)^{2} \underline{z}_{s} w_{n}$ holds. Now by Lemma 5.4 and the fact that $w_{i}$ is of maximal length in the sense that $w_{n+2}=w_{n} w_{n+1}=x\left(w_{i}\right)^{3} y$, we must have $i=n-3$ such that $\left(w_{i}\right)^{2} \leq_{s} w_{n}, w_{i} \leq_{p} w_{n+1}$ and $\left(w_{i}\right)^{3} \in E\left(w_{n+2}\right)$

(2) Suppose $w_{i} \Sigma_{p} w_{n+1}$. Then let $\left(w_{i}\right)^{r} u_{1} \leq_{s} w_{n}, u_{2}\left(w_{i}\right)^{s} \leq_{p} w_{n+1}$, where $r, s \geq$ $0, r+s=2, w_{i}=u_{1} u_{2}, u_{1}, u_{2} \in X^{+}$. It is clear that $i \leq n-1$. But $w_{n-1} \leq_{p} w_{n+1}$ and hence $i \leq n-2$. By Lemma 5.4(3), we have $\left(w_{i}\right)^{2} \leq_{s} w_{n}$.

(i) If $r \geq 1$, then $\left(w_{i}\right)^{r} u_{1} \leq_{s} w_{n}$. Thus since $\left(w_{i}\right)^{2} \leq_{s} w_{n}$ and $w_{i}=u_{1} u_{2}$, the condition $u_{2} u_{1}=u_{1} u_{2}$ holds. By Lemma $1.7([4])$, we have that $u_{1}$ and $u_{2}$ are powers of a common word and then $w_{i} \notin Q$, a contradiction.

(ii) If $r=0$, then $u_{1} \leq_{s} w_{n}$ and $u_{2}\left(w_{i}\right)^{2} \leq_{p} w_{n+1}$. Since $u_{1} \leq_{p} w_{i}$ and $u_{2} \leq_{s} w_{i}$, by Proposition 5.6, both $u_{1}$ and $u_{2}$ are in $F_{a, b}^{1}$. It follows that $u_{1}=w_{i-2}, u_{2}=w_{i-1}$. That is $w_{i-1}\left(w_{i}\right)^{2} \leq_{p} w_{n+1}$. Consider

$$
\begin{aligned}
w_{n+1} & =w_{n-1} w_{n} \\
& =w_{n-3} w_{n-2} w_{n-2} w_{n-1}
\end{aligned}
$$

and by Lemma $5.4(2), w_{n-4} \leq_{s} w_{n}$. Hence $i=n-2$ and $\left(w_{n-2}\right)^{3} \in E\left(w_{n+2}\right)$. 
By the above proposition, we see that $F_{a, b}^{1} \cap G_{3} \neq \emptyset$ and it is also true that $F_{a, b}^{0} \cap G_{3} \neq$ $\emptyset$. We now give some concrete examples as follows.

In $F_{a, b}^{0}, z_{1}=a, z_{2}=b, z_{3}=b a, z_{4}=b a b, z_{5}=b a b b a, z_{6}=b a b b a b a b, z_{7}=$ $b a b b a b a b b a b b a, z_{8}=b a b b a(b a b)(b a b)(b a b) a b b a b a b$ and $(b a b)^{3}$ is a subword of $z_{8}$.

In $F_{a, b}^{1}, w_{1}=a, w_{2}=b, w_{3}=a b, w_{4}=b a b, w_{5}=a b b a b, w_{6}=b a b a b b a b, w_{7}=$ $a b b a b b a b a b b a b, w_{8}=b a b a b b a(b a b)(b a b)(b a b) a b b a b$, and $(b a b)^{3}$ is a subword of $w_{8}$.

There is a word $u \in X^{+}, u \notin F_{a, b}^{1}$ such that $u^{3} \in E\left(w_{n}\right), w_{n} \in F_{a, b}^{1}$. This can be seen from the term,

$$
\begin{aligned}
w_{9} & =a b b a b b a b a b b(a b b a b)(a b b a b)(a b b a b) b a b a b b a b \\
& =a b b a b b a b a b(b a b b a)(b a b b a)(b a b b a) b b a b a b b a b .
\end{aligned}
$$

Where both $a b b a b a b b a b a b b a b=(a b a b b)^{3}$ and $b a b b a b a b b a b a b b a=(b a b b a)^{3}$ are subwords of $w_{9}$.

In the final part of this paper we will show that for any $u \in X^{+}, u^{4}$ is not a subword of any Fibonacci word.

Proposition 5.8. Let $w_{n} \in F_{a, b}^{1}, n \geq 1$. Then $\left(w_{i}\right)^{4} \notin E\left(w_{n}\right)$, for every word $w_{i} \in F_{a, b}^{1}, i<n$.

Proof. By observation, the proposition holds true for $n \leq 9$. We now show that the proposition is true for all $n \geq 10$. Suppose, for $n \geq 8$,

$$
w_{n+2}=w_{n} w_{n+1}=x\left(w_{i}\right)^{4} y, \text { for some } i .
$$

Then by Proposition 5.7, $i$ can not be $n-1$. Consider $i=n-2$ and $i=n-3$. By Proposition 5.7, we have that one of the following two conditions holds:

(1) If $w_{i} \leq_{p} w_{n+1}$, then $i=n-3$, i.e., $w_{n-3} \leq_{p} w_{n+1}$ and $\left(w_{n-3}\right)^{2} \leq_{s} w_{n}$. If (1) holds, then by Lemma $5.4(1),\left(w_{n-3}\right)^{2} \mathbb{Z}_{p} w_{n+1}$ and by Lemma $5.4(4),\left(w_{n-3}\right)^{3} \underline{L}_{s} w_{n}$, we have that $\left(w_{n-3}\right)^{4} \notin E\left(w_{n+2}\right)$.

(2) If $w_{i} \mathbb{z}_{p} w_{n+1}$, then $i=n-2$, i.e., $w_{n-4} \leq_{s} w_{n}$ and $w_{n-3}\left(w_{n-2}\right)^{2} \leq_{p} w_{n+1}$.

If (2) holds, then (i) we claim that $w_{n-2} w_{n-4} \mathbb{L}_{s} w_{n}$. If not, then $w_{n-2} w_{n-4}=w_{n-4} w_{n-3}$ $w_{n-4}$ and we have $w_{n-3} w_{n-4} \leq_{s} w_{n}$. But by Lemma $5.4(2), w_{n-2} \leq_{s} w_{n}$. Since $l g\left(w_{n-2}\right)=l g\left(w_{n-4} w_{n-3}\right)=l g\left(w_{n-3} w_{n-4}\right)$, we have that $w_{n-4} w_{n-3}=w_{n-3} w_{n-4}$. By Lemma $1.7([3]), w_{n-3}$ and $w_{n-4}$ are powers of a common word and then $w_{n-2} \notin Q$, a contradiction. So $w_{n-2} w_{n-4} \mathbb{L}_{s} w_{n}$. (ii) We claim that $w_{n-3}\left(w_{n-2}\right)^{3} \not_{p} w_{n+1}$. By Lemma $5.5,\left(w_{n-3} w_{n-4}\right)^{3}=w_{n-3}\left(w_{n-4} w_{n-3}\right)^{2} w_{n-4} \not_{p} w_{n+1}$ and then $w_{n-3}\left(w_{n-4} w_{n-3}\right)^{3} \underline{Z}_{p}$ $w_{n+1}$. Thus we have that $w_{n-3}\left(w_{n-2}\right)^{3}=w_{n-3}\left(w_{n-4} w_{n-3}\right)^{3} \underline{x}_{p} w_{n+1}$. By (i) and (ii), we have that $\left(w_{n-2}\right)^{4} \notin E\left(w_{n+2}\right)$.

Finally, for $i<n-3$, by the recursion of the words in $F_{a, b}^{1}$, we conclude that $\left(w_{i}\right)^{4} \notin$ $E\left(w_{n+2}\right)$ and the proof is completed.

If $w_{n}$ is the $n$-th term of $F_{a, b}^{1}, n \geq 4$, then $w_{n}$ is an overlapping word. This can be seen from the fact that

$$
w_{n}=w_{n-2} w_{n-3} w_{n-2} \text {. }
$$


Proposition 5.9. Let $w_{n} \in F_{a, b}^{1}, n \geq 1$. For $w_{n} \neq u \in X^{+}$, if $u \leq_{p} w_{n}$ and $u \leq_{s} w_{n}$, then $u \in\left\{w_{i} \mid n-2 \geq i \geq 2, n-i\right.$ is even $\}$.

Proof. Let $u \in X^{+}$. If $u \leq_{p} w_{n}$ and $u \leq_{s} w_{n}$, then, by Proposition 5.6 and Lemma 5.3 , we have $u \in\left\{w_{i} \mid n-2 \geq i \geq 2, n-i\right.$ is even $\}$.

Lemma 5.10. Let $w_{n} \in F_{a, b}^{1}, n \geq 1$. Then the following two conditions hold: (1) $w_{n} \underline{Z}_{p} w_{n+1}$ and $w_{n} \notin \bar{E}\left(w_{n+1}\right)$,

(2) for $n \geq 2, w_{n} \notin \bar{E}\left(w_{n} w_{n-1}\right)$.

Proof. (1) By observation, we see that $w_{n} \underline{\Sigma}_{p} w_{n+1}$. We now show that $w_{n} \notin$ $\bar{E}\left(w_{n+1}\right)$. Suppose $w_{n} \in \vec{E}\left(w_{n+1}\right)$. Then, for some $x, y \in X^{+}$,

$$
w_{n+1}=w_{n-1} w_{n}=x w_{n} y \text {. }
$$

Thus $l g(x)+l g(y)=l g\left(w_{n-1}\right)$ and there exist $w_{n}^{\prime}, w_{n}^{\prime \prime} \in X^{+}$such that

$$
w_{n}=w_{n}^{\prime \prime} y=w_{n}^{\prime} w_{n}^{\prime \prime}
$$

Then, by Proposition 5.9, $w_{n}^{\prime \prime} \in\left\{w_{i} \mid n-2 \geq i \geq 2, n-i\right.$ is even $\}$. Now let $w_{n}^{\prime \prime}=w_{i}$, for some $n-2 \geq i \geq 2$. Consider $w_{n}=w_{n-2} w_{n-1}=w_{n-2} w_{n-3} w_{n-2}$. If $\lg \left(w_{i}\right)=$ $l g\left(w_{n}^{\prime \prime}\right)=l g\left(w_{n-2}\right)$, then $l g(y)=l g\left(w_{n-1}\right)$. From Equation (5.4), we see that $l g(x)=0$, a contradiction. Therefore $l g\left(w_{i}\right)<l g\left(w_{n-2}\right)$. From $w_{n}=w_{n}^{\prime \prime} y=w_{i} y$, we must have $l g(y)>l g\left(w_{n-1}\right)$. This contradicts to that $l g(x)+l g(y)=l g\left(w_{n-1}\right)$. The statement (1) is then true. (2) By similar argument as in the proof of (1), we can prove (2).

Lemma 5.11. Let $w_{n} \in F_{a, b}^{1}, n \geq 1$. If for some $u \in X^{+}, u^{2} \in E\left(w_{n}\right)$, then $l g(u) \leq \lg \left(w_{n-2}\right)$. In other words, if $\lg (u)>\lg \left(w_{n-2}\right)$, then $u^{2} \notin E\left(w_{n}\right)$.

Proof. Suppose $u^{2} \in E\left(w_{n}\right)$ for some $n \geq 1$ and $l g(u)>\lg \left(w_{n-2}\right)$. Then there exist $x, y, u_{1}, u_{2}, u_{3}, u_{4} \in X^{*}$ such that

$$
w_{n}=w_{n-2} w_{n-3} w_{n-2}=x u_{1} u_{2} u_{3} u_{4} y
$$

where $u=u_{1} u_{2}=u_{3} u_{4} ; w_{n-3}=u_{2} u_{3} ; w_{n-2}=x u_{1}=u_{4} y$. Now

(1) if $\lg \left(u_{1}\right) \leq \lg \left(u_{3}\right)$, then

$$
l g(u)=l g\left(u_{1}\right)+l g\left(u_{2}\right) \leq l g\left(u_{3}\right)+l g\left(u_{2}\right)=\lg \left(w_{n-3}\right)<\lg \left(w_{n-2}\right)
$$

which is not true.

(2) If $l g\left(u_{1}\right)>\lg \left(u_{3}\right)$, then from the condition that

$$
l g(u)=l g\left(u_{3}\right)+l g\left(u_{4}\right)>l g\left(w_{n-2}\right)=l g\left(u_{4}\right)+l g(y),
$$

we see that $l g\left(u_{3}\right)>l g(y)$ and $u_{3} \in X^{+}$. Since $l g\left(u_{1}\right)>l g\left(u_{3}\right)$, there exists $z \in X^{+}$ such that

$$
u_{1}=u_{3} z, \text { and } u_{4}=z u_{2}
$$


And then

$$
w_{n-2}=x u_{3} z=z u_{2} y \text {. }
$$

Since $z \leq_{p} w_{n-2}, z \leq_{s} w_{n-2}$ and $u_{3} \in X^{+}$, then $z \neq w_{n-2}$ and then by Proposition $5.9, \lg (z) \leq l g\left(w_{n-4}\right)$. Now from $\lg \left(u_{3}\right)>\lg (y)$, we have $\lg \left(u_{2} y\right)<\lg \left(u_{2} u_{3}\right)=$ $\lg \left(w_{n-3}\right)$ and then $\lg (z)>\lg \left(w_{n-4}\right)$, a contradiction.

By (1) and (2), the result is true.

Lemma 5.12. Let $w_{n} \in F_{a, b}^{1} n \geq 1$. If $\lg (u)=l g\left(w_{n+1}\right)$, then $u^{2} \notin E\left(w_{n} w_{n+1} w_{n}\right)$. Moreover, if $u^{2} \in E\left(w_{n+1} w_{n+1} w_{n}\right)$, then $u=w_{n+1}$. That is, $u^{2} \notin \bar{E}\left(w_{n+1} w_{n+1} w_{n}\right)$.

Proof. Suppose $l g(u)=l g\left(w_{n+1}\right)$ and $u^{2} \in E\left(w_{n} w_{n+1} w_{n}\right)$. Let

$$
w_{n} w_{n+1} w_{n}=x u_{1} u_{2} u_{3} u_{4} y
$$

where $x, y, u_{1}, u_{2}, u_{3}, u_{4} \in X^{*} ; \quad u=u_{1} u_{2}=u_{3} u_{4}$ and $w_{n}=x u_{1}=u_{4} y ; \quad w_{n+1}=u_{2} u_{3}$. Since $l g(u)=l g\left(w_{n+1}\right)$, we have $l g\left(u_{1}\right)=l g\left(u_{3}\right), \lg \left(u_{2}\right)=l g\left(u_{4}\right)$. By $u_{1} \leq_{s} w_{n}, u_{3} \leq_{s}$ $w_{n+1}$, then $u_{1}=u_{3}$. But $u_{2} \leq_{p} w_{n+1}, u_{4} \leq_{p} w_{n}$, by properties of the sets $F_{1}^{1}$ and $F_{2}^{1}$, we see that $u_{2} \neq u_{4}$. It follows that $u=u_{1} u_{2} \neq u_{3} u_{4}=u$, a contradiction. The former part of the lemma is then true.

By the similar argument as in the proof of first part, we are able to show that $u^{2} \notin E\left(w_{n+1} w_{n+1} w_{n}\right)$.

Lemma 5.13. Let $w_{n} \in F_{a, b}^{1}, n \geq 1$. If $\lg \left(w_{n+1}\right)>\lg (u)>\lg \left(w_{n}\right)$, then (1) $u^{3} \notin E\left(w_{n+1} w_{n} w_{n+1} w_{n+1}\right) ;(2) u^{3} \notin E\left(w_{n+1} w_{n} w_{n+1} w_{n}\right)$. $X^{*}$,

Proof. (1) Suppose $u^{3} \in E\left(w_{n+1} w_{n} w_{n+1} w_{n+1}\right)$ and for $x, y, u_{1}, u_{2}, u_{3}, u_{4}, u_{5}, u_{6} \in$

$$
w_{n+1} w_{n} w_{n+1} w_{n+1}=x u_{1} u_{2} u_{3} u_{4} u_{5} u_{6} y
$$

where $u=u_{1} u_{2}=u_{3} u_{4}=u_{5} u_{6} ; w_{n+1}=x u_{1}=u_{4} u_{5}=u_{6} y ; w_{n}=u_{2} u_{3}$. Since $l g\left(w_{n+1}\right)>l g(u)>l g\left(w_{n}\right)$, by assumption, we have $l g\left(u_{1}\right)>l g\left(u_{3}\right), \lg \left(u_{3}\right)<\lg \left(u_{5}\right)$. Thus there exist $z_{1}, z_{2} \in X^{+}$such that $u_{1}=u_{3} z_{1}, u_{4}=z_{1} u_{2} ; u_{5}=u_{3} z_{2}, u_{4}=z_{2} u_{6}$. Hence

$$
w_{n+1}=u_{4} u_{5}=z_{1} u_{2} u_{3} z_{2}=z_{1} w_{n} z_{2} .
$$

This contradicts to Lemma 5.10(1) and condition of Lemma 5.13 (1) holds true.

(2) Similar argument as the proof of (1) will work for the proof of (2).

By the same technique used in the proof of Lemma 5.13, we are able to show the following:

Corollary 5.14 Let $w_{n} \in F_{a, b}^{1}, n \geq 1$. If $\lg \left(w_{n+1}\right)>\lg (u)>\lg \left(w_{n}\right)$, then (1) $u^{3} \notin E\left(w_{n+1} w_{n+1} w_{n} w_{n+1}\right)$; (2) $u^{3} \notin E\left(w_{n} w_{n+1} w_{n} w_{n+1}\right)$.

Proposition 5.15. Let $w_{n} \in F_{a, b}^{1}, n \geq 1$. Then $w_{n} \notin G_{4}$. 
Proof. By observation the proposition is true for $n \leq 7$. Now suppose there exists a $j \geq 1$ such that $w_{j} \in G_{4}$. Let $w_{m}$ be the first Fibonacci word in $F_{a, b}^{1} \cap G_{4}$. Then $w_{m-1}, w_{m-2} \notin G_{4}$. By Proposition 5.8, we have that, $\left(w_{i}\right)^{4} \notin E\left(w_{m}\right)$, for $w_{i} \in F_{a, b}^{1}$, $1 \leq i<m$. Thus we consider for the case $u \notin F_{a, b}^{1}$ and $u^{4} \in E\left(w_{m}\right)$. Let

$$
w_{m}=w_{m-2} w_{m-1}=x u^{4} y, \text { for some } x, y \in X^{*} .
$$

Then since, by assumption, $w_{m}$ is the first Fibonacci word in $F_{a, b}^{1} \cap G_{4}$, we have $x \leq_{p}$ $w_{m-2}, y \leq_{s} w_{m-1}$.

(A) If $\lg (u)>\lg \left(w_{m-3}\right)$, then by Lemma 5.11, $u^{2} \notin E\left(w_{m-1}\right)$ and then $u^{4} \notin E\left(w_{m}\right)$.

(B) If $\lg (u) \leq l g\left(w_{m-6}\right)$, then, by $\lg \left(w_{n}\right)=\lg \left(w_{n-2}\right)+\lg \left(w_{n-1}\right)$ and $3 \cdot \lg \left(w_{n-1}\right)<$ $2 \cdot \lg \left(w_{n}\right)$

$$
\begin{aligned}
l g\left(u^{4}\right) & \leq 4 \cdot \lg \left(w_{m-6}\right) \\
& =\lg \left(w_{m-6}\right)+3 \cdot \lg \left(w_{m-6}\right) \\
& <l g\left(w_{m-6}\right)+2 \cdot \lg \left(w_{m-5}\right) \\
& =l g\left(w_{m-4}\right)+\lg \left(w_{m-5}\right) \\
& =l g\left(w_{m-3}\right) .
\end{aligned}
$$

Thus $\lg \left(u^{4}\right)<\lg \left(w_{m-3}\right)$. Since $w_{m}=w_{m-2} w_{m-1}=w_{m-4} w_{m-3} w_{m-3} w_{m-2}$ and $u^{4}$ $\notin E\left(w_{m-2}\right), u^{4} \notin E\left(w_{m-1}\right)$, we have $u^{4} \in E\left(w_{m-3} w_{m-3}\right)$. But $w_{m-3} w_{m-3} \leq_{s} w_{m-1}$ (see Equation (5.2)), we have that $u^{4} \in E\left(w_{m-1}\right)$ and this contradict to that $w_{m}$ is, by assumption, the first Fibonacci word such that $u^{4} \in E\left(w_{m}\right)$.

By above (A) and (B), we have if $u^{4} \in E\left(w_{m}\right)$, then

$$
l g\left(w_{m-6}\right)<l g(u) \leq l g\left(w_{m-3}\right) .
$$

In order to complete the proof of that no $u \in X^{+} \backslash F_{a, b}^{1}$ such that $u^{4} \in E\left(w_{n}\right)$, for $w_{n} \in F_{a, b}^{1}$, we consider the following three equations :

$$
\begin{gathered}
w_{m}=w_{m-2} w_{m-3} w_{m-2}=w_{m-4} w_{m-3} w_{m-3} w_{m-4} w_{m-3} \\
w_{m}=w_{m-4} w_{m-5} w_{m-4} w_{m-5} w_{m-4} w_{m-4} w_{m-5} w_{m-4} \\
=w_{m-6} w_{m-7} w_{m-4} w_{m-4} w_{m-5} w_{m-4} w_{m-4} w_{m-5} w_{m-4} \\
w_{m}=w_{m-6} w_{m-5} w_{m-5} w_{m-6} w_{m-5} w_{m-5} w_{m-6} w_{m-5} w_{m-4} w_{m-3}
\end{gathered}
$$

(1) Consider $l g(u)=l g\left(w_{i}\right), i=m-3, m-4, m-5$. In each case, by Equations (5.5), (5.6) and (5.7) and looking at the length of $u$, we see that $u^{2} \in E\left(w_{i} w_{i} w_{i-1}\right)$. But by Lemma $5.12 u=w_{i}$, this contradicts to $u \notin F_{a, b}^{1}$. Thus we have $u^{2} \notin E\left(w_{i} w_{i} w_{i-1}\right)$. Hence $u^{4} \notin E\left(w_{m}\right)$.

We now consider the remaining cases:

(2) If $l g\left(w_{m-4}\right)<l g(u)<l g\left(w_{m-3}\right)$, then by Equation (5.5) we have that $u^{3} \in$ $E\left(w_{m-3} w_{m-3} w_{m-4} w_{m-3}\right)$. It contradicts to Corollary $5.14(1)$. 
(3) If $l g\left(w_{m-5}\right)<\lg (u)<\lg \left(w_{m-4}\right)$, then by Equation (5.6), we have that either (3-1) $u^{3} \in E\left(w_{m-4} w_{m-5} w_{m-4} w_{m-5}\right)$, This contradicts to Lemma $5.13(2)$; or (3-2) $u^{3} \in E\left(w_{m-4} w_{m-5} w_{m-4} w_{m-4}\right)$, this contradicts to Lemma $5.13(1)$.

(4) If $l g\left(w_{m-6}\right)<l g(u)<l g\left(w_{m-5}\right)$, then by Equation (5.7), we have that either (4-1) $u^{3} \in E\left(w_{m-5} w_{m-6} w_{m-5} w_{m-5}\right)$, this contradicts to Lemma 5.13(1); or (4-2) $u^{3} \in E\left(w_{m-5} w_{m-5} w_{m-6} w_{m-5}\right)$, this contradicts to Corollary $5.14(1)$.

By above (1), (2), (3) and (4), we complete the proof of that there is no word $u \in X^{+} \backslash F_{a, b}^{1}$ with $\lg \left(w_{m-6}\right)<\lg (u) \leq \lg \left(w_{m-3}\right)$ such that $u^{4} \in E\left(w_{m}\right)$.

The proof of the proposition is then completed.

For $u, v \in X^{+}$, we call $v$ the mirror image of $u$ if $u=a_{1} a_{2} \cdots a_{r}$, for some. $r \geq 1$ and $a_{1}, a_{2}, \ldots, a_{r} \in X$, then $v=a_{r} a_{r-1} \cdots a_{1}$. Now we can prove the following corollary.

Corollary 5.16. Let $z_{n} \in F_{a, b}^{0} n \geq 1$. Then $z_{n} \notin G_{4}$.

Proof. If $w_{n}$ is the $n$-th term of $F_{a, b}^{1}$ and $z_{n}$ is the $n$-th term of $F_{a, b}^{0}$, then $z_{n}$ is the mirror image of $w_{n}$. (This can be proved by induction on $n$ and from the fact that $w_{n}=w_{n-2} w_{n-3} w_{n-2}$ and $z_{n}=z_{n-2} z_{n-3} z_{n-2}$. ) With this fact it is clear that $z_{n} \notin G_{4}$, otherwise $w_{n}$ will be in $G_{4}$.

The following is now clear:

Proposition 5.17. The atom Fibonacci languages $F_{a, b}^{0}$ and $F_{a, b}^{1}$ are subsets of $G^{(1)} \cup G^{(2)} \cup G^{(3)}$.

\section{References}

[1] Wai-fong Chuan, Fibonacci Words, The Fibonacci Quarterly, 30(1)(1992), 68-76.

[2] A. de Luca, "A combinatorial property of Fibonacci words," Inform. Proc. Letters, 12(1981), 193195.

[3] D. E. Knuth, The Art of Computer Programming, Vol. I, New York, Addision-Wesley, 1973.

[4] H. J. Shyr, Free Monoids and Languages, Second Edition, Hon-Min Book Company, Taichung, Taiwan, 1991.

[5] H. L. Wu, On the properties of Primitive words, M.S. Thesis, Department of Mathematics, ChungYuan Christian University, Chung Li, Taiwan, 1992.

Management Information Department, National Chin-Yi Institute of Technology, Taichung, Taiwan. Institute of Applied Mathematics, National Chung-Hsing University, Taichung, Taiwan. 